\title{
A Dependence of Hadron Production in Inelastic Muon Scattering and Dimuon Production by Protons
}

\author{
S. Frankel and W. Frati \\ Physics Department, University of Pennsylvania
}

Nov. 15, 1993

\begin{abstract}
The A dependence of the production of hadrons in inelastic muon scattering and of the production of dimuons in high $Q^{2}$ proton interactions are simply related. Feynman $\mathrm{x}$ distributions and $\mathrm{z}$ scaling distributions in nuclei are compared with energy loss models. Suggestions for new data analyses are presented.

PACS numbers: 25.30.Mr 25.40.Ep
\end{abstract}




\section{Introduction}

When muons scatter from a proton or neutron the nucleon is excited and its structure is changed because of the interaction of the muon with a quark in the proton. $q \bar{q}$ pairs which eventually become on-shell pions are produced and the yield of pions can be measured as a function of $\mathrm{z}$, the ratio of the pion energy to the maximum pion energy allowable in the process.

The time evolution of this state just after the interaction is unknown but it can be studied by measuring the same reaction in nuclei. In a sense the nucleus samples the emerging hadrons because the hadrons only rarely escape the nucleus without rescattering. They impart energy to the spectator nucleons and hence lose energy themselves before coming on-shell.

The final state (fully clothed) hadrons produced in this interaction are formed roughly at the dilated time $(\nu / m)\left(r_{h} / c\right)$, where $\nu$ is the energy imparted to the nucleon and $r_{h}$ is the hadron size. For the data we study in this paper the energies, $\nu$, are in the hundreds of $\mathrm{GeV}$.

Because the hadronization products cannot be calculated by perturbative QCD we carry out a study of models of the hadronization process in reactions involving muons.

In one model the nucleon is excited and passes through the nucleus losing energy to the spectator nucleons in its path. This energy loss by the excited nucleon reduces the maximum energy available to the hadrons (mainly pions) it produces and will thus 
affect the value of $z=E_{h} / E_{\max }$. Because the muons are weakly interacting, they only interact at their full energy with a single target nucleon. (The evolution is shown in Fig. 1.)

In a second model it is possible that the $q \bar{q}$ pairs, which eventually become the pions, are produced in the initial interaction. These pairs interact with the spectator nucleons on passing through the nucleus, imparting energy to them. (The evolution is shown in Fig. 2.)

It is not difficult to estimate the effects of energy loss on either model. What is needed is an assumption about the interaction cross section. We can assume for simplicity that these off-shell hadrons have a cross section, $\sigma_{\text {inel }}$, for reinteraction that is the measured p-nucleon or pi-nucleon cross section, but we shall demonstrate how the $\mathrm{z}$ dependence depends on a range of $\sigma_{\text {inel }}$ 's. We also need an estimate of the energy loss of the hadrons per collision. With this information the nuclear z distributions can be obtained.

In a parallel study, also involving muons, but now in the final state, we examine the production of dimuons by protons: The incoming proton interacts with spectator nucleons and makes soft minimum bias collisions before making the high $Q^{2}$ dimuon pair. Thus the incoming proton's energy can be decreased, thereby reducing the energy available for producing the rare dimuon pair. This shifts events to lower Feynman x. $\mathrm{x}=\mathrm{E}($ dimuon $) / \mathrm{E}_{\max }($ dimuon$)$. (The evolution for this reaction is shown in Fig. 3.)

In muon production of hadrons the A dependence is often revealed by exam- 
ining $\mathrm{R}(\mathrm{z})=\mathrm{N}_{\mathrm{A}}(\mathrm{z}) / \mathrm{N}_{\mathrm{D}}(\mathrm{z})$ while in dimuon production by protons the quantity is $\mathrm{R}(\mathrm{x})=\mathrm{N}_{\mathrm{A}}(\mathrm{x}) / \mathrm{N}_{\mathrm{D}}(\mathrm{x}) . \quad \mathrm{N}$ is the number of events at either $\mathrm{x}$ or $\mathrm{z}$. The comparison is usually made with the deuteron, $\mathrm{D}$, to include possible $\mathrm{p}-\mathrm{n}$ differences.

A most interesting value of $\mathrm{R}$ is the limiting value at $\mathrm{z}$ or $\mathrm{x}$ equal to unity. In the hadron production case, if the the muon interacts with the last nucleon in its path, there will be no energy loss. Only such events will appear at $\mathrm{z}=1$. In the dimuon case, the events at $\mathrm{x}=1$ arise from those interactions where the incident proton made the dimuon on the first nucleon in its path, the weakly interacting dimuon pair passing unscathed through the nucleus. If $a_{n}$ is the (Glauber) probability of making $\mathrm{n}$ collisions in a proton-nucleon minimum bias interaction in a nucleus, $n a_{n}$ is the probability of making a rare high $Q^{2}$ collision like a muon scatter or the production of a dimuon pair or vector meson such as a $J / \Psi$. Since the probability of making one collision is then $n a_{n} / n=a_{n}$ the fraction of times the collision takes place in a first or last collision is just $\sum a_{n} / \sum n a_{n}=1 /<n>$ Thus these endpoints depend only on Glauber probabilities and not on the detailed mechanisms. This general argument shows that $\mathrm{R}$ cannot be a constant, independent of $\mathrm{z}$ or $\mathrm{x}$. It is not difficult to calculate these endpoint values using a Woods-Saxon spatial distribution for the nucleons and the total inelastic cross section for the nucleon-nucleon scattering. That endpoint does not depend on the energy loss function or its magnitude.

Actually, we really need to know the inelastic cross section for an off-shell hadron on a ground state nucleon if we wish to calculate $\mathrm{R}$ at any other value than $\mathrm{R}(1)$. 


\section{Hadron Production in Inelastic Muon Interactions}

Figure 4 shows a plot of $\mathrm{R}(\mathrm{z})$ taken from the data of ref. [1] The authors have chosen to separate their data sample into a low $Q^{2}$ - low $x_{B j}$ bin and a high $Q^{2}$ - high $x_{B j}$ bin, where $Q^{2}$ is the four momentum transfer to the nucleon and $x_{B j}$ is Bjorken x. As these authors have shown, and because it can be seen that the measured points are almost identical in the two samples, there is apparently no nuclear dependence in the data on these variables. As a result, and to improve the statistical accuracy of our comparison, we have suitably averaged the two results which are shown plotted in Figure 5 .

To examine the relative merits of fits of the data to the conclusion in ref. [1] that there is no A dependence, we have examined the relative $\chi^{2}$ for a linear fit hypothesis to the data shown in Figure 5 . For the fit to $\mathrm{R}=1$, the $\chi^{2}$ for the five measured points is 7.2. It is 3.0 for the best linear energy loss fit shown in the figure. (Eliminating the low statistics high z point hardly changes the slope of the falloff but improves the $\chi^{2}$ by 2 rather than 1 unit.) The authors of ref. [1] remark that there are large systematic

errors in the lowest $\mathrm{z}$ point. Without that point we find that the $\chi^{2}$ is .53 for the linear fit and 3.2 for a flat fit with no A dependence, i.e. $\mathrm{R}=1$. Thus an A dependent effect seems to provide a better fit to the data, suggesting that there is a nuclear effect to be understood.

In several previous papers we have examined the effects of energy loss in nuclear 
interactions. [2] [3] [4] The calculation of $\mathrm{R}(\mathrm{z})$ is simple if one knows the form of the energy loss per collision. Various models [5] [6] have been proposed recently, giving different assumptions for the $\sqrt{s}$ dependence of the energy loss. We make use of the model we used in 1987 [3] since it was demonstrated to be in agreement with the data observed in low $p_{t}$ production and was the functional form for the energy loss per nucleon obtained by examining the energy loss found in the ISAJET minimum bias model of F. Paige [7], widely used by particle physicists. That energy loss is approximately given by $d \sqrt{s} / d n=$ constant $=\beta$, where $\mathrm{n}$ is the number of collisions. Thus in our model the energy loss varies as the square root of the laboratory energy, $\sqrt{E}$ This energy dependence lies between the values $E^{0}$ and $E^{1}$ of refs. [5] and [6] respectively.

We first consider the model of the struck proton hadronizing outside the nucleus into pions. In Fig. 6 we have plotted our results for several values of $\nu=\mathrm{E}_{\mu}-\mathrm{E}_{\mu^{\prime}}$. After these calculations were made we obtained the actual $\nu$ distributions obtained in the experiment. That average value is $\nu=170 \mathrm{GeV}$ which one can see from Figure 6 would fall well on the experimental results. All the theoretical curves predict a rise above $\mathrm{R}=1$ at low $\mathrm{z}$ due to the sliding back of events to lower $\mathrm{z}$ due to energy loss. While this appears in the data, the authors of ref. 1 caution that there are large systematic errors in the lowest $\mathrm{z}$ point. Note that the point at $\mathrm{z}=.68$ falls well below $\mathrm{R}=1$. For this calculation we used $d \sqrt{s} / d n=.2 \mathrm{GeV}$, which we had found in earlier work fit the available data on dimuon and $J / \Psi$ production $[4$. The theoretical curves 
have been corrected to take into account the bin size used in the data. The $\mathrm{N}(\mathrm{z})$ data for both Xe and D were fitted to a sum of two exponentials of the form $e^{-\alpha z}$ to get the best representation of the input to the energy loss calculation. To show the sensitivity of the calculations to both the inelastic cross section, $\sigma_{\text {inel }}$, and $d \sqrt{s} / d n$, we show in Figures 7 and 8 how variation in these parameters affects the results. These plots also show the asymptotic limits. There is roughly a trade-off of $d \sqrt{s} / d n$ of $.1 \mathrm{GeV}$ for a change in the inelastic cross section of $10 \mathrm{mbs}$.

We now turn to the second calculation, namely the model in which the $q \bar{q}$ pairs are formed at the collision and lose energy on the way out of the nucleus. Fig. 9 shows our results for an inelastic pion-nucleon cross section of $20 \mathrm{mb}$ and an energy loss $d \sqrt{s} / d n$ $=.2$ for different values of the energy of the pion. The dependence on energy is small and all curves show a depression below $\mathrm{R}=1$. There is a good fit to the data if we again omit the lowest $\mathrm{z}$ point as suggested in ref. [1]. Figure 10 shows the effect of varying the energy loss when $\nu=170 \mathrm{GeV}$ and $\sigma_{\text {tot }}=20 \mathrm{mbs}$.

Fig 11 shows the effect of varying the cross section of the pion using the average value of $\nu$ of $170 \mathrm{GeV}$ and the same energy loss parameter. Here we see that $20 \mathrm{mb}$ appears to give the better fit than 10 or $31 \mathrm{mb}$. Once again we note different limits at $\mathrm{R}=1$

Comparing Figs 9, 10, and 11 with Figs 5, 6 and 7, we note that in the case of the pion energy loss the slopes of $\mathrm{R}$ vs $\mathrm{z}$ are smaller and the main effect is a depression of $\mathrm{R}$ below unity. 
We conclude that while the data are not very precise they do not rule out the presence of energy loss mechanisms, as suggested by W. Busza [9].

\section{Dimuon Production in p-A Collisions}

Dimuon production in p-A collisions can be calculated on the same energy loss model, but is slightly more complicated since the dimuon production cross section is energy dependent and the shape of the cross section depends on the invariant mass of the dimuon pair.

The effect on $\mathrm{R}\left(x_{F}\right)=\sigma_{h-A}\left(x_{F}\right) / A \sigma_{h-p}\left(x_{F}\right)$ of any loss in energy comes about because of the $\sqrt{s}$ dependence of the Drell-Yan cross section which varies as $e^{\gamma M / \sqrt{s}}$. $M$ is the invariant mass of the muon pair. The energy loss therefore produces two effects a) a reduction in the dimuon yield and b) a displacement of events to lower $x_{F}$. Before demonstrating the effects of this energy loss on $\mathrm{R}\left(x_{F}\right)$ we present a "back of the envelope" calculation of $\mathrm{R}=\sigma_{p-A} / A \sigma_{p-p}$, which shows the qualitative features.

1) $R=1-(\gamma)(M / \sqrt{s})(\beta / \sqrt{s})(\langle n\rangle-1)$

This formula demonstrates how the various parameters enter into the dimuon "depression". ( Empirically the last bracket in eq. 1) varies very roughly as ln A so, for small departures from unity, $\mathrm{R}$ will vary as $\mathrm{A}$ raised to a small constant.) While $\mathrm{R}$ should approach unity at large s, [ $[$ it will not be unity at lab energies as high as 800 $\mathrm{GeV}$, nor will $\mathrm{R}\left(x_{F}\right)$ be independent of $x_{F}$. 
We now turn to two pieces of data that illustrate the effect of energy loss. We use the Drell-Yan formalism and the Duke-Owens form factors for the calculation [10], rather than using the empirical values for the energy dependence of the cross section, since we have checked that they give essentially the same results.

Fig. 12 shows the data of the NA10 collaboration on dimuon production by 140 and $286 \mathrm{GeV}$ pions [11]. Superimposed on their data are our calculations for .2 and .4 $\mathrm{GeV}$ energy loss. There appears to be a clear $\mathrm{M}$ dependence as well as deviations from $\mathrm{R}=1$ in the $x_{F}$ distributions. The data is not very precise but the general features of energy loss are borne out.

Fig. 13 shows the $800 \mathrm{GeV}$ data 12] of Fermilab expt. E 772. We have obtained the unpublished mass distributions [13] for each $x_{F}$ bin so we can make a comparison between the data for different values of $d \sqrt{s} / d n$. The published $x_{F}$ distributions for their $\mathrm{W}$ to Deuterium ratios show a deviation in $\mathrm{R}$ from unity at large $x_{F}$. Our calculations for $d \sqrt{s} / d n=0.2$ are superimposed on the data.

We conclude that there are A dependent effects in dimuon production which can be accounted for using the same energy loss mechanism that can account for the A dependence in inelastic muon scattering and using the same approximate energy loss parameter.

\section{Discussion}

Unfortunately, none of the data extend to very large values of $\mathrm{z}$ or $\mathrm{x}$ to enable the 
asymptotic values at $R(1)$ to be compared with the Glauber prediction, verifying the importance of the energy loss in the most unambiguous way. There are other recent estimates for the functional form of the energy loss [5] [6] but the present data cannot easily be used to discriminate among the various models.

However the question of the form of the energy dependence of $d \sqrt{s} / d n$ can be more easily examined with present data on hadron production by muons. E665 can use all its data not just their published low and high $Q^{2}$ - Bjorken x bins to improve the statistical accuracy for a new study: the separation of the present E 665 muon inelastic data into separate $\nu$ bins to study $\mathrm{R}(\nu)$. Newer E665 data could be analyzed to study $\mathrm{R}$ for a wide range of A. For example, with our parameterization $d \sqrt{s} / d n$, the laboratory energy loss, $\mathrm{dE} / \mathrm{dn}$, would vary as $\sqrt{E}$ so that the loss parameter would clearly change in the region covered by the E665 data (110 to $490 \mathrm{GeV}$ ). While it is difficult to study the energy dependence of $d \sqrt{s} / d n$ in dimuon production, since different accelerator energies are needed, it is simple to select $\nu$ from the inelastic muon data to make the same study.

This work extends to muon inelastic scattering and dimuon production the need for energy loss in traversing nuclear matter in accounting for A dependences which we showed earlier [3] could account for various A dependences in minimum bias reactions.

\section{Acknowledgements}

We should like to thank J. Ryan and W. Busza for useful and spirited discussions. 


\section{Figures}

Fig. 1 Hadron Production by Muons: An incident muon interacts with a nucleon which rescatters off another nucleon in the nucleus. The nucleon struck by the muon hadronizes outside the nucleus.

Fig. 2 Pion Production by Muons: An incident muon interacts with a nucleon producing an off-shell pion which rescatters off nucleons in the nucleus

Fig. 3 Dimuon Production by Hadrons: An incident proton makes soft nonperturbative scatters before making a high $Q^{2}$ collision with another nucleon producing an excited proton which hadronizes outside the nucleus.

Fig. 4 The $\mathrm{z}$ dependence of the ratio of hadron production in Xenon and Deuterium, $\mathrm{R}(X e / D)$, for high (open circles) and low (solid circles) $Q^{2}$ and $x_{B j}$. The data are taken from ref. 1. Table XXXI.

Fig. $5 \mathrm{R}$ vs $\mathrm{z}$ for the combined sample of high and low $Q^{2}$ data. The solid curve is a least-square fit to a straight line fit to the data.

Fig. 6 Hadron Energy Loss: $\mathrm{R}$ vs $\mathrm{z}$ for the combined sample of high and low $Q^{2}$ data. Theoretical curves are shown for three values of the energy transfer, $\nu$. The mean value of $\nu$ for the data is $170 \mathrm{GeV}$.

Fig. 7 Hadron Energy Loss: 7 Theoretical calculations of $\mathrm{R}$ for different values of 
$d \sqrt{s} / d n$. The inelastic $\mathrm{p}-\mathrm{p}$ cross section is set at $31 \mathrm{mb}$. The value of $\mathrm{R}$ at $\mathrm{z}=1$ is set by this cross section and the Woods-Saxon nucleon spatial distribution.

Fig. 8 Hadron Energy Loss: Calculations of $\mathrm{R}$ for different inelastic cross sections. The value of $d \sqrt{s} / d n=.2 \mathrm{GeV}$. Note the different asymptotes.

Fig. 9 "Pion Energy Loss": $\mathrm{R}$ vs z for the combined sample of high and low $Q^{2}$ data. Theoretical curves are shown for three values of the energy transfer, $\nu$. The pion-nucleon cross section is taken as $20 \mathrm{mb} . d \sqrt{s} / d n=.2$. The mean value of $\nu$ for the data is $170 \mathrm{GeV}$.

Fig. 10 "Pion" Energy Loss: Theoretical calculations of $\mathrm{R}$ for different values of $d \sqrt{s} / d n=.1, .2, .3$, and .4 . The inelastic pi-p cross section is set at $20 \mathrm{mb}$. The value of $R$ at $z=1$ is set by this cross section and the Woods-Saxon nucleon spatial distribution.

Fig. 11 "Pion" Energy Loss: Calculations of R for different inelastic cross sections, 10, 20 and $31 \mathrm{mb}$. The value of $d \sqrt{s} / d n=.2 \mathrm{GeV} . \nu=170 \mathrm{GeV}$. Note the different asymptotes.

Fig. 12 Data of ref. [11] for dimuon production by pions. The predicted falloff of $\mathrm{R}$ with the dimuon invariant mass, $\mathrm{M}$, as well as with Feynman $\mathrm{X}$ are shown along with the theoretical energy loss predictions.

Fig. 13 Dimuon production by $800 \mathrm{GeV}$ protons. $\mathrm{R}$ vs $x_{F}$. Data from ref. 12 


\section{References}

[1] M. R. Adams et al., Phys. Rev. D 5 Aug 1 (1994) (E665)

[2] The basic ideas in this note appeared in " $J / \Psi$ and Dimuon Production in Ultrarelativistic Nuclear Collisions" Quark Matter in Collision, World Scientific, 1988, P. Carruthers and J. Rafelski, eds., pp 565-7 and were amplified in "Dimuon and Vector Meson Production in Nuclei", University of Pennsylvania, UPR-0423T, May 5, 1990.

[3] S. Frankel and W. Frati, Physics Letters B196 (1987) 399 ; Nucl. Phys. B308 (1988) 699.

[4] S. Frankel and W. Frati, Z. Phys. C 57 (1993) 225

[5] S. Brodsky, Nucl. Phys. A544 223 (1992).

[6] E. Quack, "Parton Initial State Scattering ...", Heidelberg preprint HD - TVP $92-2$.

[7] Frank Paige, BNL Report 30805, Jan. 1982

[8] Geoffrey T. Bodwin, Stanley J. Brodsky, and G. Peter Lepage, Phys. Rev. Lett. 471799 (1981).

[9] Wit Busza, Nucl. Phys. A544 49c (1992)

[10] D. Duke and J. Owens, Phys. Rev.D30 (1984) 49. 
[11] P. Bordalo et al. Phys. Lett. B193 369 (1987).

[12] D. M. Alde et al., Phys. Rev. Lett. 64 (1981) 2479.

[13] J.C. Peng, private communication

muhad4ds.tex 8-94 
This figure "fig1-1.png" is available in "png" format from: http://arxiv.org/ps/hep-ph/9410239v1 
This figure "fig2-1.png" is available in "png" format from: http://arxiv.org/ps/hep-ph/9410239v1 
This figure "fig3-1.png" is available in "png" format from: http://arxiv.org/ps/hep-ph/9410239v1 
This figure "fig4-1.png" is available in "png" format from: http://arxiv.org/ps/hep-ph/9410239v1 
This figure "fig5-1.png" is available in "png" format from: http://arxiv.org/ps/hep-ph/9410239v1 
This figure "fig1-2.png" is available in "png" format from: http://arxiv.org/ps/hep-ph/9410239v1 
This figure "fig2-2.png" is available in "png" format from: http://arxiv.org/ps/hep-ph/9410239v1 
This figure "fig3-2.png" is available in "png" format from: http://arxiv.org/ps/hep-ph/9410239v1 
This figure "fig4-2.png" is available in "png" format from: http://arxiv.org/ps/hep-ph/9410239v1 
This figure "fig5-2.png" is available in "png" format from: http://arxiv.org/ps/hep-ph/9410239v1 
This figure "fig3-3.png" is available in "png" format from: http://arxiv.org/ps/hep-ph/9410239v1 
This figure "fig4-3.png" is available in "png" format from: http://arxiv.org/ps/hep-ph/9410239v1 
This figure "fig5-3.png" is available in "png" format from: http://arxiv.org/ps/hep-ph/9410239v1 